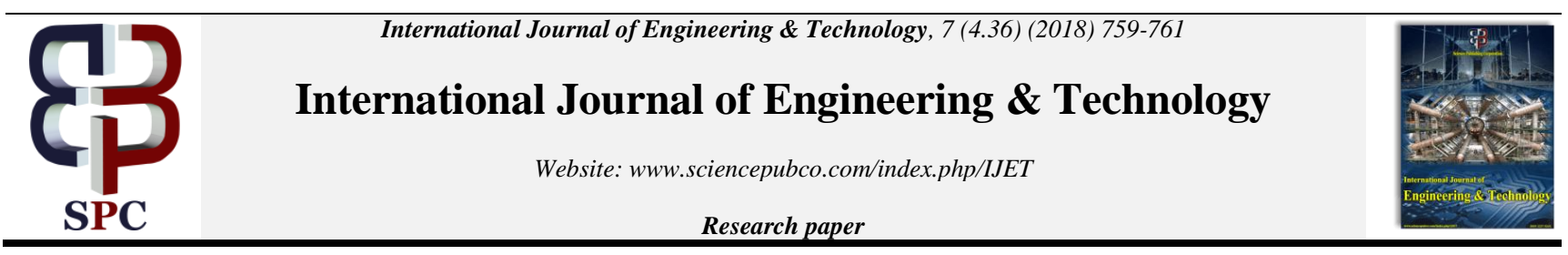

\title{
Coefficient Inequality for Certain New Subclasses of Sakaguchi Type Function Related to Sigmoid Functions
}

\author{
B. Srutha Keerthi ${ }^{*}$, Bhuvaneswari Raja ${ }^{2}$ \\ ${ }^{1}$ Department of Mathematics, School of Advanced Sciences, VIT Chennai, Vandaloor, Kelambakam Road, Chennai, India. \\ ${ }^{2}$ Research Scholar, Department of Mathematics, Bharathiar University, Coimbatore, India. \\ E-mail:shri_bhuva@yahoo.co.in \\ *Corresponding author E-mail: sruthilaya06@yahoo.co.in
}

\begin{abstract}
The question of the present paper is to get starting coefficients ||$a_{2}|,| a_{3}|,| a_{4} \mid$, upper limits of $a_{3}-\mu a_{2}^{2} \mid$ and second Hankel determinant related with a class of systematic univalent capacity of sakaguchi compose work identified with sigmoid capacity in the open unit plate $\Delta$. Different creators as Abiodum, Tinuoye Oladipo, Murugu sundaramoorthy et. al., and Olatunji have contemplated sigmoid capacity for various classes of systematic and univalent capacities. Our outcomes fills in as a speculation toward this path and it conceives an offspring some current subclasses of capacities.
\end{abstract}

Keywords: Analytic function, coefficient estimate, Starlike function, subordination, Convex function, univalent function, upper bound, sigmoid function, Differential operator, Second Hankel determinant.

\section{Introduction}

The hypothesis of unique capacity has over sparkling by different fields like genuine investigation, practical examination, topology, polynomial math, differential conditions and soon. The summed up hyper geometric capacities assumes a noteworthy job in geometric capacity hypothesis after the confirmation of Bieberbach Guess by de-Branges. Despite the fact that the unique capacities does not have a particular definition, its application generally stretch out to material science, PC and so on.

There are different uncommon capacities however we will worry with one of the actuation work known as sigmoid capacity or straightforward calculated capacity. It is more well known as a result of its angle descendent learning calculation. Sigmoid capacity is the most usually known capacity utilized in feed forward neural systems on account of its non-linearity and the computational straightforwardness of its subordinate.

Actuation work is an in data process comprising of countless handling elements(neurons), propelled by a similar way organic apprehensive system(such as cerebrum), cooperating to comprehend a particular undertaking. The capacity can be learned by model, yet can't be customized to do particular undertakings. It tends to be assessed in various ways, most uncommonly by truncated arrangement development. This capacity can be arranged into three, to be specific, incline work, edge work and sigmoid capacity. The sigmoid capacity of the frame is differentiable and has the accompanying properties:

$$
h(z)=\frac{1}{1+e^{-x}}
$$

- It outputs real numbers between 0 and 1 .

- It maps a very large input domain to a small range of outputs.

- It never loses information because it is an injective function.

- It increases monotonically.

Sigmoid function is perfectly useful in geometric function theory with all the four properties.

Let Abe the class of all univalent analytic functions $f$ of the for

$$
f(z)=z+\sum_{k=2}^{s} a_{k} z^{k}
$$

defined in the open unit disk $\Delta=\{z: z \in \mathrm{C}$ and $|z|<1\}$, and normalized by the conditions $f(0)=0$ and $f^{\prime}(0)=1$ for $f \in A$. Recall that $S^{*}$ and $C$ denotes the class of star like and convex functions which their geometric condition satisfies 
$R e\left\{\frac{z f^{\prime}(z)}{f(z)}\right\}>0$ and $R e\left\{1+\frac{z f^{\prime \prime}(z)}{f^{\prime}(z)}\right\}>0, z \in \Delta$. Several authors have used the above two classes of functions in different ways of perspectives.

An analytic function $f$ is subordinate to an analytic function $g$, written $f(z) \prec g(z)$, if there is an analytic function $w: \Delta \rightarrow \Delta$ with $w(0)=0$ satisfying $f(z)=g(w(z))$. It follows from Schwarz lemma that $f(z)<g(z)(z \in \Delta) \Rightarrow f(0)=g(0)$ and

$f(\Delta) \subset g(\Delta)$. If $g(z)$ is univalent, then $f(z) \prec g(z),(z \in \Delta) \leftrightarrow f(0)=$ $g(0)$ and $f(\Delta) \subset g(\Delta)$.

Let $P$ be the class of Caratheodory function with positive real part consisting of all analytic functions $p: \Delta \rightarrow C$ satisfying $p(0)=1$ and $\operatorname{Rep}(\bar{z})>0$.

We need the following results about the functions belonging to the class $P$.

If the function $p \in P$ is given by the series

$$
p(z)=1+c_{1} z+c_{2} z^{2}+c_{3} z^{3}+\ldots
$$

then the following sharp estimates holds;

$$
\left|c_{n}\right| \leq 2(n=1,2,3, \ldots) \text {. }
$$

In 1976, Noonan and Tomas [5] stated the $q^{m}$ Hankel determinant for $q \geq 1$ and $n \geq 1$ are defined by

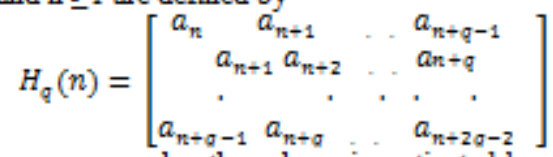

In recent years, several authors have investigated bounds for the Hankel determinant of functions belonging to various subclasses of univalent and multivalent functions. The Hankel determinant $H_{2}(1)=a_{3}-a_{2}^{2}$ is the well knownFekete-Szegö functional. For results related to this functional, see. The second Hankel determinant $H_{2}(2)$ is given by $H_{2}(2)=a_{2} a_{4}-a_{3}^{2}$.

For the purpose of our results, the following lemma shall be necessary.

Lemma 1.1. Let $\mathrm{h}$ be a sigmoid function and

$$
\phi(\mathrm{z})=2 \mathrm{~h}(\mathrm{z})=1+\sum_{m=1}^{\infty} \frac{(-1)^{n}}{\mathrm{z}^{m}}\left(\sum_{n=1}^{\infty} \frac{(-1)^{n}}{n !} \mathrm{z}^{n}\right)^{m}
$$

then $\phi(\mathrm{z}) \in \mathrm{P},|\mathrm{z}|<1$ where $\phi(\mathrm{z})$ is modified sigmoid function Lemma 1.2. Let $h$ be a sigmoid function and

$$
\phi_{m, n}(\mathrm{z})=2 \mathrm{~h}(\mathrm{z})=1+\sum_{n=1}^{\infty} \frac{(-1)^{n}}{\mathrm{z}^{m}}\left(\sum_{n=1}^{\infty} \frac{(-1)^{n}}{n !} \mathrm{z}^{n}\right)^{m}
$$

then $\left|\phi_{m, n}(\mathrm{z})\right|<2$.

Lemma 1.3 If $\phi(\mathrm{z}) \in \mathrm{P}$ and it is starlike, then $f$ is normalised univalent function of the form (1.2), taking $m=1$, Fadipe et al. remarked that

$$
\phi(\mathrm{z})=1+\sum_{n=1}^{\infty} c_{n} z^{n}
$$

wherec $n=\frac{(-1)^{n+1}}{2 n !}$ then $\left|c_{n}\right| \leq 2,(n=1,2,3, \ldots)$, the result is sharp for each $n$

Definition 1.4. Let the class $L^{\lambda, n}\left(\tau, \phi_{m, n}\right), \tau \in \backslash C\{0\}$ denote the subclass of A consisting of functions $f$ of the form (1.2) satisfying

$$
R e\left[1+\frac{1}{\tau}\left[\frac{(1-t)\left[x^{2}\left(D_{n-1} f(x)\right)+r\left(D_{n-1} f(x)\right)\right]}{\lambda x\left[\left(D_{n-1} f(x)\right)-t\left(D_{n-1} f(t r)\right)\right]+(1-\lambda)\left[D_{n-1} f(x)-D_{n-1} f(t r)\right]}-1\right]\right]>0,
$$

for $0 \leq \lambda \leq 1,|t| \leq 1, t \neq 1$ and $\phi_{m, n}(z)$ is a simple logistic sigmoid activation function [9] and

when $\mathrm{n}+1>0$.

\section{Coefficient estimates}

Various authors as Abiodum [6], Timuoye Oladipo[3], Murugusundaramoorthy et. al.[4], and Olatunji $[7,8]$ have studied sigmoid function for different classes of analytic and univalent functions. In this paper, we obtain few coefficient bounds for the class

Theorem 2.1. If $f(z)$ given by (1.2) belongs to the class $L^{\lambda, n}\left(\tau, \phi_{m, n}\right), m \geq 2$ then

$$
\begin{aligned}
& \left|a_{z}\right| \leq \frac{|z|}{z(n+2)(2+\lambda) /(2-t)}, \\
& \left|a_{z}\right| \leq \frac{(1+t)|z|^{2}}{z(n+1)(n+z)(2+z) / 2-t)\left(z-t-t^{2}\right)}
\end{aligned}
$$

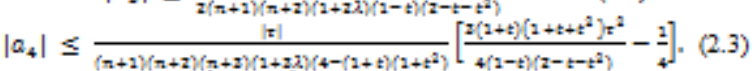
Proof. If $f(z) \in L^{\lambda, n}\left(\tau, \phi_{m, n}\right)$ then

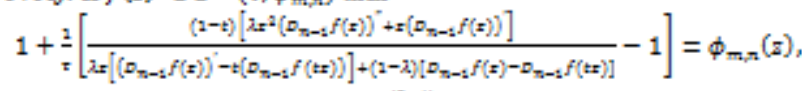
(2.4)

Where Taylor's series expansion of $\phi_{m, n}(z)$ gives

$$
\phi_{m, n}(z)=1+\frac{1}{z} z-\frac{1}{24} z^{2}+\frac{1}{240} z^{2}-\frac{17}{40270} z^{7}+\ldots
$$

from (2.4) we have

$$
\begin{aligned}
& 1 \frac{1}{\tau}\left[(n+1)(1+\lambda)(1-t) a_{2} z\right. \\
& +\frac{1}{2}[(n+1)(n+2)(1+2 \lambda) \\
& +\left[\frac{1}{6}(n+1)(n+2)(n+3)(1+3 \lambda)\left(3-t-t^{2}-t^{3}\right) a_{4}\right. \\
& +\frac{1}{2}\left[( n + 1 ) ^ { 2 } ( n + 2 ) ( 1 + \lambda ) ( 1 + 2 \lambda ) \left(-3-t+2 t^{2}\right.\right. \\
& \left.\left.+2 t^{3}\right)\right] a_{2} a_{3}+(n+1)^{3}(1+\lambda)^{3}(1+t)^{2}(1 \\
& \left.\left.-t) a_{2}^{3}\right] z^{3}+\ldots\right]=\phi_{m, n}(z) .
\end{aligned}
$$

Equating the coefficients of $z, z^{2}$ and $z^{3}$, we obtain

$$
\begin{gathered}
a_{2} \leq \frac{\tau}{2(n+1)(1+\lambda)(1-t)}, \\
a_{3} \leq \frac{(1+t) \tau^{2}}{2(n+1)(n+2)(1+2 \lambda)(1-t)\left(2-t-t^{2}\right)},(2.7) \\
a_{4} \leq \frac{\tau}{(n+1)(n+2)(n+3)(1+3 \lambda)\left(3-t-t^{2}-t^{3}\right)}\left[\frac{3(1+t)\left(1+t+t^{2}\right) \tau^{2}}{4(1-t)\left(2-t-t^{2}\right)}-\frac{1}{4}\right] .
\end{gathered}
$$

Results (2.1),(2.2) and (2.3) can be obtained from (2.6), (2.7) and (2.8) respectively.

Corollary 2.2.If $f(z) \in S(\tau, \phi)$, then [10]

$\left|a_{2}\right| \leq \frac{|\tau|}{2(n+1)}$

$\left|a_{3}\right| \leq \frac{|\tau|^{2}}{4(n+1)(n+2)}$

$\left|a_{4}\right| \leq \frac{|\tau|}{3(n+1)(n+2)(n+3)}\left[\frac{3 \tau^{2}}{8}-\frac{1}{4}\right]$.

Corollary 2.3.If $f(z) \in C(\tau, \phi)$, then [10]

$$
\begin{aligned}
\left|a_{2}\right| & \leq \frac{|\tau|}{4(n+1)}, \\
\left|a_{3}\right| & \leq \frac{|\tau|^{2}}{12(n+1)(n+2)^{\prime}} \\
\left|a_{4}\right| & \leq \frac{|\tau|}{12(n+1)(n+2)(n+3)}\left[\frac{3 \tau^{2}}{8}-\frac{1}{4}\right] .
\end{aligned}
$$

\section{Fekete-Szegö inequalities}

Recently there has been interest to obtain the Fekete-Szegö inequality for various subclasses of $\mathrm{S}$ and $\mathrm{C}$. In this section making use of $a_{2}$ and $a_{3}$, we prove the following Fekete-Szegö result for the function class $L^{\lambda, n}\left(\tau, \phi_{m, n}\right)$.

Theorem 3.1.If $f(z)$ belongs to the class $L^{\lambda, n}\left(\tau, \phi_{m, n}\right)$, of the form (1.7), then 
$\left|a_{3}-\mu a_{2}^{2}\right| \leq \frac{|\tau|^{2}}{4(n+1)^{2}(1+\lambda)^{2}(1-t)^{2}}\left[\frac{2\left(1-t^{2}\right)(1+\lambda)^{2}(n+1)}{(n+2)(1+2 \lambda)\left(2-t-t^{2}\right)}-\mu\right]$.

Proof. From (2.6) and (2.7)

$a_{3}-\mu a_{2}^{2} \leq \frac{|\tau|^{2}}{4(n+1)^{2}(1+\lambda)^{2}(1-t)^{2}}\left[\frac{2\left(1-t^{2}\right)(1+\lambda)^{2}(n+1)}{(n+2)(1+2 \lambda)\left(2-t-t^{2}\right)}-\mu\right]$

hence (3.1) can be easily obtained from (3.2).

Corollary 3.2.If $f(z) \in S(\tau, \phi)$, then [10]

$\left|a_{3}-\mu a_{2}^{2}\right| \leq \frac{|\tau|^{2}}{4(n+1)^{2}}\left[\frac{(n+1)}{(n+2)}-\mu\right]$

Corollary 3.3.If $f(z) \in C(\tau, \phi)$, then [10]

$\left|a_{3}-\mu a_{2}^{2}\right| \leq \frac{|\tau|^{2}}{16(n+1)^{2}}\left[\frac{4(n+1)}{3(n+2)}-\mu\right]$

\section{Second Hankel determinant}

In this section making use of $a_{2}$ and $a_{3}$, we obtain the following second Hankel determinant result for the function class $L^{\lambda, n}\left(\tau, \phi_{m, n}\right)$

Theorem 4.lif $f(z) \in L^{\lambda, n}\left(\tau, \phi_{m, n}\right)$, then

$$
\left|\begin{array}{c}
\left|a_{2} a_{4}-a_{3}^{2}\right| \leq \\
\frac{\tau^{2}}{8(n+1)^{2}(n+2)(n+3)(1+\lambda)(1+3 \lambda)(1-t)\left(3-t-t^{2}-t^{2}\right)} \\
{\left[-1+\frac{3 t^{2}(1+t)\left(1+t+t^{2}\right)}{(1-t)\left(2-t-t^{2}\right)}-\frac{2 \tau^{2}(1+t)^{2}(1+\lambda)(1+3 \lambda)(n+3)\left(3-t-t^{2}-t^{2}\right)}{(n+2)(1+2 \lambda)^{2}\left(2-t-t^{2}\right)^{2}(1-t)}\right]}
\end{array}\right|
$$

Proof. From (2.6), (2.7) and (2.8), we have

$$
\begin{gathered}
\left(a_{2} a_{4}-a_{3}^{2}\right) \leq \\
\frac{\tau^{2}}{s(n+1)^{2}(n+2)(n+3)(1+\lambda)(1+3 \lambda)(1-t)\left(3-t-t^{2}-t^{2}\right)} \\
{\left[-1+\frac{3 t^{2}(1+t)\left(1+t+t^{2}\right)}{(1-t)\left(2-t-t^{2}\right)}-\frac{2 \tau^{2}(1+t)^{2}(1+\lambda)(1+3 \lambda)(n+3)\left(3-t-t^{2}-t^{2}\right)}{(n+2)(1+2 \lambda)^{2}\left(2-t-t^{2}\right)^{2}(1-t)}\right]}
\end{gathered}
$$

(4.2)

which gives the desired inequality (4.1)

Corollary 4.2.If $f(z) \in S(\tau, \phi)$, then [10]

$$
\left|a_{2} a_{4}-a_{3}^{2}\right| \leq\left|\frac{\tau^{2}}{24(n+1)^{2}(n+2)(n+3)}\left[-1+\frac{3 \tau^{2}}{2}-\frac{3 \tau^{2}(n+3)}{2(n+2)}\right]\right| \text {. }
$$

Corollary 4.3.If $f(z) \in C(\tau, \phi)$, then [10]

$$
\left|a_{2} a_{4}-a_{3}^{2}\right| \leq\left|\frac{\tau^{2}}{192(n+1)^{2}(n+2)(n+3)}\left[-1+\frac{3 \tau^{2}}{2}-\frac{4 \tau^{2}(n+3)}{3(n+2)}\right]\right|
$$

\section{Conclusion}

By selecting the values of $\lambda$ and $t$ we state the interesting FeketeSzegöinequality and Second Hankel determinant for the subclasses of $C(\tau ; \varphi)[1,2]$.The results above serve as a new generalisation of subclasses of univalent functions related to sigmoid functions. The investigation of initial coefficients bounds, Fekete-Szegöinequality and Second Hankel determinant for various subclasses can be a scope of future research.

\section{References}

[1] Ali RM, Ravichandran V \& Seenivasagan N, "Coefficient bounds for p-valent functions", Applied Mathematics and Computation, Vol.187, No.1, (2007), pp.35-46.

[2] Ali RM, Ravichandran V \& Lee SK, "Subclasses of multivalent starlike and convex functions", Bulletin of the Belgian Mathematical Society-Simon Stevin, Vol.16, No.3, (2009), pp.385-394.

[3] Fadipe-Joseph, OA, Oladipo, AT \& Ezeafulukwe, UA, "Modified sigmoid function in univalent function theory", International Journal of Mathematical Sciences and Engineering Application, Vol.7, No.7, (2013), pp.313-317.

[4] Murugusundaramoorthy G \& Janani T, "Sigmoid Function in the Space of Univalent \$Lambda \$-Pseudo Starlike Functions",
International Journal of Pure and Applied Mathematics, Vol.101, No.1, (2015), pp.33-41.

[5] Noonan JW \& Thomas DK, "On the second Hankel determinant of areally mean $p$-valent functions", Transactions of the American Mathematical Society, Vol.223, (1976), pp.337-346.

[6] Oladipo AT, "Coefficient inequality for subclass of analytic univalent functions related to simple logistic activation functions", Stud. Univ. Babes-Bolyai Math, Vol.61, (2016), pp.45-52.

[7] Olatunji SO, "Sigmoid function in the space of univalent lambda pseudo starlike function with Sakaguchi type functions", Journal of Progressive Research in Mathematics, Vol.7, No.4, (2016), pp.1164-1172.

[8] Sharma, P \& Raina, RK, "On a Sakaguchi type class of analytic functions associated with quasi-subordination", Rikkyo Daigaku sugaku zasshi, Vol.64, No.1, (2015), pp.59-70.

[9] Sakar FM, Aytas S and Guney O, "On the Fekete-Szeg" $o$ problem for a generalised class defined by differential operator", Suleyman Derminal University Journal of Natural and Applied Sciences, Vol.20, No.3, (2016), pp.456-459.

[10] Altınkaya S,, "Application of Quasi-Subordination for Generalized Sakaguchi Type Functions”, Journal of Complex Analysis, (2017). 